\title{
Approximation of nonconvex distributed parameter optimal control problems
}

\author{
I. Chryssoverghi \\ Department of Mathematics, National Technical University \\ Zografou Campus, 15773 Athens, Greece. \\ Phone: 301-7721716. Fax: 301-7721775. \\ E-mail: ichriso@nisyros.ntua.gr
}

\begin{abstract}
We consider a nonconvex optimal control problem involving nonlinear parabolic systems with several equality and inequality state constraints. Existence and extremality results are obtained using relaxation theory. We then completely discretize the problem and study the limit behaviour of the approximations. Finally, we propose a mixed FrankWolfe penalty method using relaxed controls.
\end{abstract}

\section{Keywords}

Optimal control, nonlinear parabolic systems, nonconvexity, relaxed controls, discretization, mixed Frank-Wolfe penalty method

\section{INTRODUCTION}

It is well known that optimal control problems have no classical solutions in general. In order to prove the existence of optimal controls, some convexity assumptions are usually made on data. In the absence of convexity, the problem is replaced by a generalized, or relaxed, one. Using relaxation theory, one can prove not only the existence of optimal controls for the relaxed problem, but also derive necessary conditions for optimality and develop approximation and optimization methods, for lumped and as well as for distributed parameter systems (see References and bibliography there).

Here we consider an optimal control problem for semilinear parabolic systems with several control and state constraints. We first establish existence and necessary conditions for optimality for the relaxed problem. We then discretize the problem by using a finite element method in space and a semi-implicit finite difference scheme in time, while the controls are independently approximated by piecewise constant relaxed or classical ones and the state constraints slightly perturbed for admissibility reasons. It turns out that the properties of discrete optimality and extremality carry over in the limit to the respective continuous properties. The above results extend those of Chryssoverghi and 
Bacopoulos (1993). In addition, we propose a mixed Frank-Wolfe penalty method, which constructs Gamkrelidze relaxed controls, for solving theoretically the continuous relaxed problem and practically the discrete relaxed problem. Finally, using a simple procedure, relaxed controls thus constructed can be approximately simulated by classical controls. A numerical example is given.

\section{THE CONTINUOUS OPTIMAL CONTROL PROBLEMS}

Let $\Omega \subset \mathbb{R}^{d}$ be a bounded domain, $I:=(0, T), T<\infty, U \subset \mathbb{R}^{d^{\prime}}$ a compact set, and $A(t)$ a second order linear elliptic differential operator. The continuous classical optimal control (CCP) is the following. The state equation is

$y_{t}+A(t) y=f(x, t, y(x, t), w(x, t))$ in $Q:=\Omega \times I$,

$y=0$ in $\partial \Omega \times I, y(x, 0)=y^{0}(x)$ in $\Omega$.

Defining the functionals

$J_{m}(w):=\int_{\Omega} g_{m}(x, t, y(x, t), w(x, t)) d x d t, 0 \leq m \leq q$,

the constraints are

$w(x, t) \in U$ in $Q, J_{m}(w)=0,1 \leq m \leq p, J_{m}(w) \leq 0, p<m \leq q$,

and the cost functional to minimize is $J_{0}(w)$.

The CCP has no classical solutions in general, and to prove the existence of optimal controls some convexity assumptions are usually made on the data. For example, if there are no state constraints, it is assumed that the set

$S(x, t, y):=\left\{(\nu, c) \mid \nu=f(x, t, y, u), u \in U, g_{0}(x, t, y, u) \leq c\right\}$

is convex, for every $(x, t, y) \in Q \times \mathbb{R}$.

In the absence of convexity, we generalize the control problem as follows. Let

$W:=\{w: \bar{Q} \longrightarrow U$ measurable $\}$

be the set of classical controls. The set of relaxed controls is defined by

$R:=\left\{r: \bar{Q} \longrightarrow M_{1}(U)\right.$ measurable $\}$

where $M_{1}(U) \subset C(U)^{*}$ is the set of probability measures on $U$ with the relative weak star topology. The set $R$ is a convex, metrizable and compact subset of $L^{1}(\bar{Q}, C(U))^{*}$ for the relative weak star topology (see Warga, 1972). Moreover, $W$ is embedded in $R$ and $W$ is dense in $R$. For $r \in \operatorname{span}(R)$ and $\phi \in L^{1}(\bar{Q}, C(U))^{*}$, we write

$\phi(x, t, r(x, t)):=\int_{U} \phi(x, t, u) r(x, t)(d u)$. 
Now set $V:=H_{0}^{1}(\Omega)$, and let $a(t, \cdot, \cdot)$ be the bilinear form on $V$ associated to $A(t)$. The continuous relaxed problem (CRP) is the following. For every $r \in R$, the state $y=y_{r}$ is the unique solution of

$\left\langle y_{t}, \nu>+a(t, y, \nu)=\int_{\Omega} f(x, t, y(x, t), r(x, t)) \nu(x) d x\right.$, for every $\nu \in V$,

$y(0)=y^{0} \in L^{2}(\Omega)($ or $V), y \in L^{2}(I, V), y_{t} \in L^{2}\left(I, V^{*}\right)$

The constraints are

$r \in R, J_{m}(r)=0,1 \leq m \leq p, J_{m}(r) \leq 0, p<m \leq q$,

and the cost functional to minimize is $J_{0}(r)$.

We suppose that the functions $f, g_{m}, 0 \leq m \leq p$, are defined on $Q \times \mathbb{R} \times U$, measurable for fixed $y, u$, continuous for fixed $x, t$, and satisfy

$|f(x, t, y, u)| \leq \phi(x, t)+b|y|$

$\left|f\left(x, t, y_{1}, u\right)-f\left(x, t, y_{2}, u\right)\right| \leq L\left|y_{1}-y_{2}\right|$,

$\left|g_{m}(x, t, y, u)\right| \leq \psi_{m}(x, t)+c_{m} y^{2}$

on $Q \times \mathbb{R} \times U$, for some $\phi \in L^{2}(Q), \psi_{m} \in L^{1}(Q)$.

Theorem 1 If there exists an admissible control, then there exists an optimal control for the CRP.

Next, we suppose in addition that the functions $g_{m y}$ are defined on $Q \times \mathbb{R} \times U$, measurable for fixed $y, u$, continuous for fixed $x, t$, and satisfy

$\left|g_{m y}(x, t, y, u)\right| \leq \xi_{m}(x, t)+c_{m}^{\prime}|y|$, in $Q \times \mathbb{R} \times U$, for some $\xi_{m} \in L^{2}(Q)$.

Define the general Hamiltonian (we drop the index $m$ )

$H(x, t, y, z, u):=z f(x, t, y, u)+g(x, t, y, u)$,

and the general adjoint equation

$-<z_{t}, \nu>+a(t, \nu, z)=\int_{\Omega}\left[f_{y}(y, r) z+g_{y}(y, r)\right] \nu d x$, for every $\nu \in V$,

$z(T)=0, z \in L^{2}(I, V), z_{t} \in L^{2}\left(I, V^{*}\right), y=y_{r}$

The directional derivative of $J$ is then given by

$D J\left(r, r^{\prime}-r\right)=\int_{Q} H\left(x, t, y, z, r^{\prime}-r\right) d x d t$. 
Theorem 2 Relaxed Minimum Principle of Optimality If $r$ is optimal for either the CRP or the CCP, then $r$ is extremal, i.e. there exist multipliers $\lambda_{m} \in \mathbb{R}, 0 \leq m \leq p$, with $\lambda_{0} \geq 0, \lambda_{m} \geq 0, p<m \leq q$, and $\sum_{0}^{q}\left|\lambda_{m}\right|=1$, such that

$H(x, t, y(x, t), z(x, t), r(x, t))=\min _{u \in U} H(x, t, y(x, t), z(x, t), u)$ a.e. in $Q$,

where $g, g_{y}$ are replaced by $\sum_{0}^{q} \lambda_{m} g_{m}, \sum_{0}^{q} \lambda_{m} g_{m y}$ in $H, z$,

$\lambda_{m} J_{m}(r)=0, p<m \leq q$.

Further results

Suppose in addition that $f=f_{1}(y)+f_{2}(u)$, with $f_{1}$ affine, $f_{2}$ nonlinear, $g_{m}=g_{1 m}(y)+$ $g_{2 m}(u)$, with $g_{1 m}$ affine, $g_{2 m}$ nonlinear, for $1 \leq m \leq p$, and $g_{1 m}$ convex, $g_{2 m}$ nonlinear, for $m=0, p<m \leq q$. If $r \in R$ (resp. $r \in W$ ) is extremal, with some $\lambda_{0}>0$, then $r$ is optimal for the CRP (resp. the CRP and the CCP).

Since $W \subset R$, in general we have

$\min J_{0}(r) \leq \inf J(w)$,

and it can be proved that the equality holds if (i) there are no state constraints, or (ii) the CCP has a solution but has no admissible abnormal (i.e. with some $\lambda_{0}=0$ ) extremal controls.

\section{DISCRETIZATION}

For every $n$, let $\left\{S_{i}^{n}\right\}_{1}^{M(n)}$ be an admissible regular triangulation of $\bar{\Omega}$ into $d$-simplices, $\left\{I_{j}^{n}\right\}_{0}^{N(n)-1}$ a subdivision of $\bar{I}:=[0, T]$ into intervals of length $\Delta t^{n}=T / N(n) \rightarrow 0$, as $n \rightarrow \infty$, and $\left\{B_{k}^{n}\right\}_{1}^{P(n)}$ a partition of $\bar{\Omega}$ into Borel subsets, with $\max _{k} \operatorname{diam}\left(B_{k}^{n}\right) \rightarrow 0$ as $n \rightarrow \infty$. Let $V^{n} \subset V$ be the set of continuous functions on $\Omega$ which are affine on each $S_{i}^{n}$. Let $R^{n} \subset R$ be the set of relaxed controls which are constant on each $B_{k}^{n}$ (discrete relaxed controls), and $W^{n} \subset W$ the set of classical controls which are constant on each $B_{k}^{n}$ (discrete classical controls). Define the discrete state equations

$$
\begin{aligned}
& \left(y_{j+1}^{n}-y_{j}^{n}, \nu\right) / \Delta t^{n}+a\left(t_{j+1}, y_{j+1}^{n}, \nu\right)=\left(f_{j}^{n}, \nu\right), \text { for every } \nu \in V^{n}, 0 \leq j \leq N-1, \\
& y_{0}^{n} \rightarrow y^{0} \text { in } V \text { as } n \rightarrow \infty, y_{j}^{n} \in V^{n}, 0 \leq j \leq N \\
& f_{j}^{n}(x):=\left(1 / \Delta t^{n}\right) \int_{I_{j}^{n}} f\left(x, t, y_{j}^{n}(x), r^{n}(x, t)\right) d t
\end{aligned}
$$

the discrete functionals

$$
J_{m}^{n}\left(r^{n}\right):=\int_{Q} g_{m}\left(x, t, y_{-}^{n}(x, t), r^{n}(x, t)\right) d x d t
$$


where $y_{-}^{n}:=y_{j}^{n}$ in $I_{j}^{n}, 0 \leq j \leq N-1$, the constraints

$r^{n} \in R^{n}$, either (1) $\left|J_{m}^{n}\left(r^{n}\right)\right| \leq \varepsilon_{m}^{n}, 1 \leq m \leq p$, or (2) $J_{m}^{n}\left(r^{n}\right)=\varepsilon_{m}^{n}, 1 \leq m \leq p$,

$J_{m}^{n}\left(r^{n}\right) \leq \varepsilon_{m}^{n}, \varepsilon_{m}^{n} \geq 0, p<m \leq q$,

where $\varepsilon_{m}^{n} \rightarrow 0$ as $n \rightarrow \infty, 1 \leq m \leq q$, and the cost functional to minimize $J_{0}^{n}\left(r^{n}\right)$. The discrete relaxed problem $D R P_{1}^{n}$ (resp. $D R P_{2}^{n}$ ) is the above problem, case (1) (resp. case (2)). The discrete classical problems $D C P_{1}^{n}, D C P_{2}^{n}$ are the problems $D R P_{1}^{n}, D R P_{2}^{n}$, where the constraint $r^{n} \in R^{n}$ is replaced by $r^{n} \in W^{n}$.

Theorem 3 If there exists an admissible control, then there exists an optimal control for the above discrete problems.

Now define the discrete adjoint equations

$$
\begin{aligned}
& \left(z_{j}^{n}-z_{j+1}^{n}, \nu\right) / \Delta t^{n}+a\left(t_{j}, \nu, z_{j}^{n}\right)=\left(f_{y j}^{n} z_{j+1}^{n}+g_{y j}^{n}, \nu\right), \text { for every } \nu \in V^{n}, 0 \leq j \leq N-1 \\
& z_{N}^{n}=0, z_{j}^{n} \in V^{n}, 0 \leq j \leq N \\
& f_{y j}^{n}(x):=\left(1 / \Delta t^{n}\right) \int_{I_{j}^{n}} f_{y}\left(x, t, y_{j}^{n}(x), r^{n}(x, t)\right) d t \\
& g_{y j}^{n}(x):=\left(1 / \Delta t^{n}\right) \int_{I_{j}^{n}} g_{y}\left(x, t, y_{j}^{n}(x), r^{n}(x, t)\right) d t
\end{aligned}
$$

Theorem 4 Discrete Relaxed Minimum Principle

If $r^{n}$ is optimal for the $D R P_{2}^{n}$, then $r^{n}$ is extremal, i.e. there exist multipliers $\lambda_{m}^{n} \in \mathbb{R}$, $0 \leq m \leq q$, with $\lambda_{0}^{n} \geq 0, \lambda_{0}^{n} \geq 0, p<m \leq q$, and $\sum_{0}^{q}\left|\lambda_{m}^{n}\right|=1$, such that

$\int_{B_{k}^{n}} H\left(x, t, y_{-}^{n}, z_{+}^{n}, r^{n}\right) d x d t=\min _{u \in U} \int_{B_{k}^{n}} H\left(x, t, y_{-}^{n}, z_{+}^{n}, u\right) d x d t, k=1, \ldots, P$,

where $z_{+}^{n}:=z_{j+1}^{n}$ on $I_{j}^{n}, 0 \leq j \leq N-1$, and $g, g_{y}$ are replaced by $\sum_{0}^{q} \lambda_{m}^{n} g_{m}, \sum_{0}^{q} \lambda_{m}^{n} g_{m y}$,

$\lambda_{m}^{n}\left[J_{m}^{n}(r)-\varepsilon_{m}^{n}\right]=0, p<m \leq q$.

Note that the minimum principle does not necessarily hold for the $D C P_{2}^{n}$ without some additional convexity or similar assumptions.

The next two theorems show the behaviour in the limit of the above discretizations.

Theorem 5 For each $n$, let $r^{n}$ be optimal for the DRP $P_{1}^{n}$ (resp. DCP $\left.P_{1}^{n}\right)$. Under appropriate feasibility assumptions on the $\varepsilon_{m}^{n}$ (see Chryssoverghi and Kokkinis, 1994), every accumulation point of the sequence $\left\{r^{n}\right\}$ is optimal for the CRP.

Theorem 6 For each $n$, let $r^{n}$ be an admissible and extremal for the DRP $P_{2}^{n}$. Under minimum feasibility assumptions on the $\varepsilon_{m}^{n}$, every accumulation point of the sequence $\left\{r^{n}\right\}$ is admissible and extremal for the CRP. 


\section{A MIXED FRANK-WOLFE PENALTY METHOD}

The method is theoretically applied to the CRP and practically to the $D R P_{2}^{n}$. Let $s_{m}>1$, $1 \leq m \leq q$, be chosen numbers, $\left\{M_{m}^{k}\right\}, 1 \leq m \leq q,\left\{\delta^{k}\right\}$, chosen sequences such that $M_{m}^{\bar{k}} \rightarrow \infty, 1 \leq m \leq q, \delta^{k} \rightarrow 0$, as $k \rightarrow \infty$, and for each $k$, define the penalized cost functional (we drop here the index $n$ )

$$
G^{k}(r):=J_{0}(r)+\sum_{1}^{p} M_{m}^{k}\left|J_{m}(r)\right|^{s_{m}}+\sum_{p+1}^{q} M_{m}^{k}\left[\max \left(0, J_{m}(r)\right)\right]^{s_{m}} \text {. }
$$

\section{Algorithm}

Choose $r_{1} \in W$.

Step 1. Set $\overline{r_{0}}:=r_{1}, l=1, k=1$.

Step 2. Find $r_{1} \in W$ such that

$$
\gamma_{l}:=D G^{k}\left(r_{l}, \overline{r_{l}}-r_{l}\right)=\min _{\bar{r} \in R} D G^{k}\left(r_{l}, \bar{r}-r_{l}\right),\left(\gamma_{l} \leq 0\right) .
$$

Step 3. If $\gamma_{l}<-\delta^{k}$, go to step 4. Else, set $r^{k}:=r_{l}, \gamma^{k}:=\gamma_{l}, k:=k+1$, and go to step 2. Step 4 . Find $\alpha_{l} \in[0,1]$ such that

$$
G^{k}\left(r_{l}+\alpha_{l}\left(\overline{r_{l}}-r_{l}\right)\right)=\min _{0 \leq \alpha \leq 1} G^{k}\left(r_{l}+\alpha\left(\overline{r_{l}}-r_{l}\right)\right)
$$

Step 5. Set $r_{l+1}:=r_{l}+\alpha_{l}\left(\overline{r_{l}}-r_{l}\right), l:=l+1$, and go to step 2 .

The classical control $\bar{r}_{l}$ is in fact computed in step 2 by piecewise minimizing a Hamiltonian, and $r_{l}$ is a convex combination of the classical controls $\overline{r_{0}}, \ldots, \overline{r_{l-1}}$ (Gamkrelidze relaxed control). Let $\left\{r^{k}:=r_{l(k)}\right\}$ be the sequence generated by the algorithm (step 3 ), and define the sequences of multipliers

$$
\begin{aligned}
& \lambda_{m}^{k}:=M_{m}^{k}\left|J_{m}\left(r^{k}\right)\right|^{s_{m}-1}, 1 \leq m \leq p, \\
& \lambda_{m}^{k}:=M_{m}^{k}\left[\max \left(0, J_{m}\left(r^{k}\right)\right)\right]^{s_{m}-1}, p<m \leq q .
\end{aligned}
$$

Theorem 7 Let $\left\{r^{k}\right\}$ be a subsequence converging to some $r \in R$ (or $R^{n}$ ).

(a) If the sequences $\left\{\lambda_{m}^{k}\right\}, 1 \leq m \leq q$, are bounded, then $r$ is admissible and extremal.

(b) Suppose that the problem has no admissible abnormal (i.e. with some $\lambda_{0}=0$ ) extremal controls. If $r$ is admissible, then the sequences $\left\{\lambda_{m}^{k}\right\}$ are bounded and $r$ is extremal.

\section{APPROXIMATION BY CLASSICAL CONTROLS}

For simplicity take $B_{k}^{n}:=S_{i}^{n} \times I_{j}^{n}$, and let $r_{l}:=\sum_{0}^{l-1} \beta_{\mu} \overline{r_{\mu}}$ be a Gamkrelidze control computed by the algorithm. Subdivide each $I_{j}^{n}$ into intervals $I_{l \mu}^{n}, 0 \leq \mu \leq l-1$, whose 
lengths are proportional to the $\beta_{l}$. The control $r^{k}$ is then approximately simulated by the classical control

$w_{l}(x, t):=\overline{r_{\mu}}(x, t)$ in $\Omega^{n} \times I_{l \mu}^{n}, 0 \leq \mu \leq l-1,0 \leq j \leq N-1$.

\section{NUMERICAL EXAMPLE}

State equation:

$y_{t}-y_{x x}=-\sin y+6 w-3$ in $Q:=(0, \pi) \times(0,1)$,

$y(0, t)=y(\pi, t)=0$ in $(0,1)$,

$y(x, 0)=0$ in $(0, \pi)$,

Constraints:

$w(x, t) \in\{0,1\}$ (two values) in $Q$,

$J_{1}(w):=\int_{Q} y(x, t) d x d t=0$

$y(x, t) \leq 0.4$ in $Q \Leftrightarrow J_{2}(w):=\int_{Q}[\max (0, y(x, t)-0.4)]^{1.4} d x d t=0$,

Cost functional:

$J_{0}(w):=\int_{Q}[y(x, t)-t \sin x]^{2} d x d t$.

After 24 iterations (in l) of the algorithm, and with $\Delta x=\pi / 40, \Delta t=1 / 40$, we found the following approximate functional values

$J_{0}=0.187101, \quad J_{1}=0.00258348, \quad J_{2}=0.000178508$.

\section{REFERENCES}

Chryssoverghi, I. and Bacopoulos, A. (1993) Approximation of relaxed nonlinear parabolic optimal control problems, Journal of Optimization Theory and Applications, 77, 31-50.

Chryssoverghi, I. and Kokkinis, B. (1994) Discretization of nonlinear elliptic optimal control problems, Systems and Control Letters, 22, 227-234.

Warga, J. (1972) Optimal control of differential and functional equations, Academic Press, New York.

Warga, J. (1977) Steepest descent with relaxed controls, Journal of Optimization Theory and Applications, 15, 674-682. 